\title{
2D Monte Carlo Simulation of Hole and Electron Transport in Strained Si ${ }^{*}$
}

\author{
GABRIELE F. FORMICONE, DRAGICA VASILESKA and DAVID K. FERRY \\ Center for Solid State Electronics Research, Arizona State University Tempe, Az, 85287-6206, USA
}

2D Monte Carlo simulations are used to study the transport properties of holes and electrons in a strained Si layer grown in the (001) direction on a $\mathrm{Si}_{1-\mathrm{x}} \mathrm{Ge}_{\mathrm{x}}$ substrate. Scattering rates are evaluated taking into account the details of the wavefunction through a self-consistent solution of the SchödingerPoisson problem. The physical model employed for the conduction band takes into account the energy splitting of the six valleys, while for the valence band we calculate the in-plane and perpendicular effective masses employing a $\mathbf{k} \cdot \mathbf{p}$ and strain Hamiltonian. Spin-orbit interaction is also taken into account. Results show that the electron mobility enhancement ratio saturates at a Ge concentration of $0.15-0.20$, while hole mobilities increase almost linearly with composition. An electric field computed by Medici simulation for nMOSFET $\mathrm{Si}_{1-\mathrm{x}} \mathrm{Ge}_{\mathrm{x}}$ based devices is also used in a bulk Monte Carlo simulator (quasi_2D Monte Carlo), to study the electron drift velocity profile vs. the distance along the channel.

Strained silicon-based devices are becoming more important as candidates to replace Si-based devices in VLSI technology due to two main reasons. First, both hole and electron mobilities increase with respect to standard Si devices. In contrast, only the hole mobility increases in a $\mathrm{Si}_{1-\mathrm{x}} \mathrm{Ge}_{\mathrm{x}}$-channel devices. Second, the same circuit configuration as in standard Si technology can be used. In the present paper, we investigate the two-dimensional (2D), low-field transport properties of electrons and holes in a strained-Si channel. Detailed descriptions of the conduction band are given by Rieger and Vogl [1], while we employ a $a b$ initio a $\mathbf{k} \cdot \mathbf{p}$, spin-orbit and strain Hamiltonian to calculate the in-plane and perpendicular effective masses. Using this data, we solve the SchrödingerPoisson problem and calculate the $2 \mathrm{D}$ scattering rates for different scattering processes. Finally, we extract low-field drift mobilities from Monte Carlo simulations.

Silicon and Germanium have different bulk lattice constants: $\mathrm{a}_{0}(\mathrm{Si})=5.43 \mathrm{~A}$ and $\mathrm{a}_{0}(\mathrm{Ge})=5.65 \mathrm{~A}$. Because of this, growing a thin Si layer on a thick $\mathrm{Si}_{1-\mathrm{x}} \mathrm{Ge}_{\mathrm{x}}$ substrate results in a strain induced in the $\mathrm{Si}$ layer itself. The lateral (in-plane) lattice constant $a_{\|}$of the strained Si layer is equal to the bulk lattice constant $\mathrm{a}_{0}(\mathrm{x})$ of the unstrained substrate, while the perpendicular (to the interface) lattice constant $a_{\perp}$ is given by:

$$
a_{\perp}=a_{0}(S i)\left[1-2 \frac{c_{12}(S i)}{c_{11}(S i)}\left(\frac{a_{\|}-a_{0}(S i)}{a_{0}(S i)}\right)\right]
$$

where $c_{12}(\mathrm{Si})=0.650 \mathrm{Mbar}$ and $\mathrm{c}_{11}(\mathrm{Si})=1.675 \mathrm{Mbar}$ [1].

The strain arising from the mismatch in the lattice constant does not affect the electron effective masses, i.e. $m_{1}=0.91 m_{0}$ and $m_{t}=0.19 m_{0}$ at $T=300 \mathrm{~K}$. However, the strain removes the degeneracy of the six $\triangle$ valleys splitting them into two separate bands: a two-

\footnotetext{
* Work supported by Motorola
} 


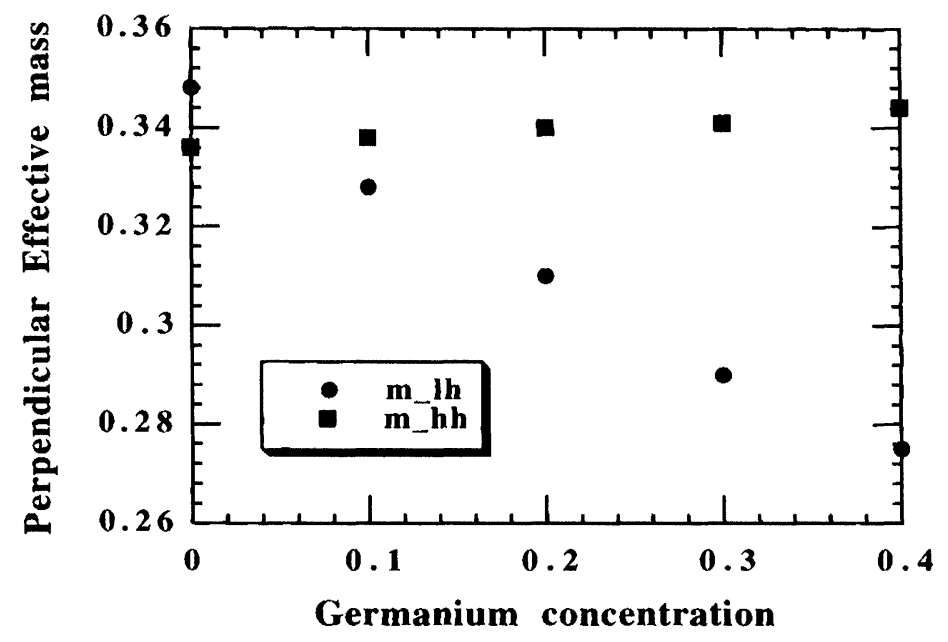

FIGURE 1 Perpendicular (longitudinal) effective masses for light and heavy holes in a strained Si channel versus Ge concentration in the substrate [9]

fold degenerate band $\Delta_{2}$ perpendicular to the interface and a four-fold degenerate band $\Delta_{4}$ parallel to the interface (i.e. in-plane). The energy splitting between the two sets of valleys is $\Delta \mathrm{E}_{\mathrm{split}}^{\mathrm{c}}=0.67 \cdot \mathrm{x}$ where $\mathrm{x}$ is the Ge concentration in the substrate [1]. The valence band structure, on the contrary, is greatly affected by the strain in terms of major changes in the effective masses and removal of the heavy- and lighthole band degeneracy at $\mathbf{k}=0$. The total Hamiltonian can be expressed as the sum of two terms ([2],[3],[4]): the strain and unstrained (spin-orbit and $\mathbf{k} \cdot \mathbf{p}$ ) Hamiltonian: $\mathrm{H}_{\mathrm{T}}=\mathrm{H}_{\text {str }}+\mathrm{H}_{\text {unstr }}$. For silicon, the valence band parameter are found to be: $\mathrm{L}=-6.64$, $\mathrm{M}=-4.60, \mathrm{~N}=-8.68, \triangle=0.044 \mathrm{eV}$ and $\mathrm{b}=2.33$ $\mathrm{eV}$. The splitting energy between the light and heavy hole bands is given by $\Delta \mathrm{E}_{\mathrm{split}}^{\mathrm{v}}=0.3953 \cdot \mathrm{x}$.

Along the direction perpendicular to the interface the (longitudinal) effective mass is:

$$
\mathrm{m}_{\mathrm{l}}\left(\mathrm{k}_{\mathrm{z}}\right)=\hbar^{2} \frac{1}{\partial^{2} \mathrm{E}(\mathrm{k}) / \partial \mathrm{k}^{2}}
$$

We chose $\mathrm{k}_{\mathrm{z}}=2.3 \cdot 10^{7} \mathrm{~cm}$ that corresponds to a perpendicular electric field of about $0.2 \mathrm{MV} / \mathrm{cm}$. The values are given in Fig. 1. At the interface, $\mathrm{k}_{\mathrm{z}}=0$, $\mathrm{k}_{\mathrm{X}}=\mathrm{k} \cos (\phi), \mathrm{k}_{\mathrm{Y}}=\mathrm{k} \sin (\phi)$ and the transverse (or parallel) effective mass is defined as:

$$
\mathrm{m}_{\perp}(\mathrm{k})=\int_{0}^{2 \pi} \mathrm{d} \phi \frac{\hbar^{2}}{2 \pi}\left|\frac{1}{\partial^{2} \mathrm{E}(\mathrm{k}, \phi) / \partial \mathrm{k}^{2}}\right|
$$

This depends on the energy and can be fitted with the following formula :

$$
m_{\perp}(E)=m_{0}+m_{1} \cdot \frac{E}{\alpha \cdot E+\beta} .
$$

The parameters $m_{0}, m_{1}, \alpha$ and $\beta$ depend upon the strain (see Table I).

The energy band model that we assume in the Monte Carlo simulator consists of two sets of equivalent valleys either for electrons or holes [5]. We take into account Coulomb, surface-roughness and phonon scattering. Coulomb scattering takes into account three different sources: depletion layer, interface-trap and oxide charges. Phonon scattering causes three different types of processes [6]: transitions between states within a single valley via acoustic phonons (i.e. intravalley acoustic-phonon scattering) and nonpolar optical phonons (i.e. intravalley optical-phonon scattering), and transitions between different valleys via nonpolar optical phonons (i.e. intervalley scattering). The intravalley acoustic-phonon scattering involves phonons with low energies and is almost an elastic process. The intra-valence band optical-phonon scattering is induced by TO phonons of low momentum 
TABLE I Parameters to fit the light and hole effective masses in a 2D strained Si layer

\begin{tabular}{cllll}
\hline$x$ & $m_{0}$ & $m_{l}$ & $\alpha$ & $\beta$ \\
\hline light hole & & & & 0.05 \\
0.0 & 0.169 & 0.291 & 2.3244 & 0.6725 \\
0.1 & 0.261 & 0.291 & 9.1242 & 0.1876 \\
0.2 & 0.261 & 0.291 & 9.7430 & 0.1028 \\
0.3 & 0.261 & 0.291 & 9.5514 & 0.4288 \\
0.4 & 0.261 & 0.291 & 9.3520 & 0.0827 \\
heavy hole & & & 0.0534 \\
0.0 & 0.433 & 1.145 & 1.5909 & 1.3843 \\
0.1 & 0.320 & 1.146 & 1.5047 & 0.0852 \\
0.2 & 0.387 & 1.145 & 1.5858 & 0.1244 \\
0.4 & 0.425 & 1.145 & 1.4288 & 0.0632 \\
\hline
\end{tabular}

and high energy. The intervalley scattering can be induced by the emission and absorption of highmomentum and high-energy phonons which can either be of acoustic or optical mode nature.

Simulations show (Fig. 2) that the low-field electron mobility increases with $\mathrm{Ge}$ concentration and, for about $\mathrm{x}=0.3$ it saturates at a ratio of 1.8 with respect to unstrained $\mathrm{Si}$. These results agree with experimental data. The same kind of behavior is obtained in the bulk (i.e. in three-dimensional-transport) [7]. This is due to the energy split in the conduction band. In fact, as $\Delta \mathrm{E}_{\mathrm{split}}^{\mathrm{c}}$ increases, intervalley phonon scattering between the two sets of valleys is reduced (even though intervalley scattering between valleys in the same set is still active). When $\Delta \mathrm{E}_{\text {split }}^{\mathrm{c}}$ is much larger than the optical phonon energy involved in this proc-

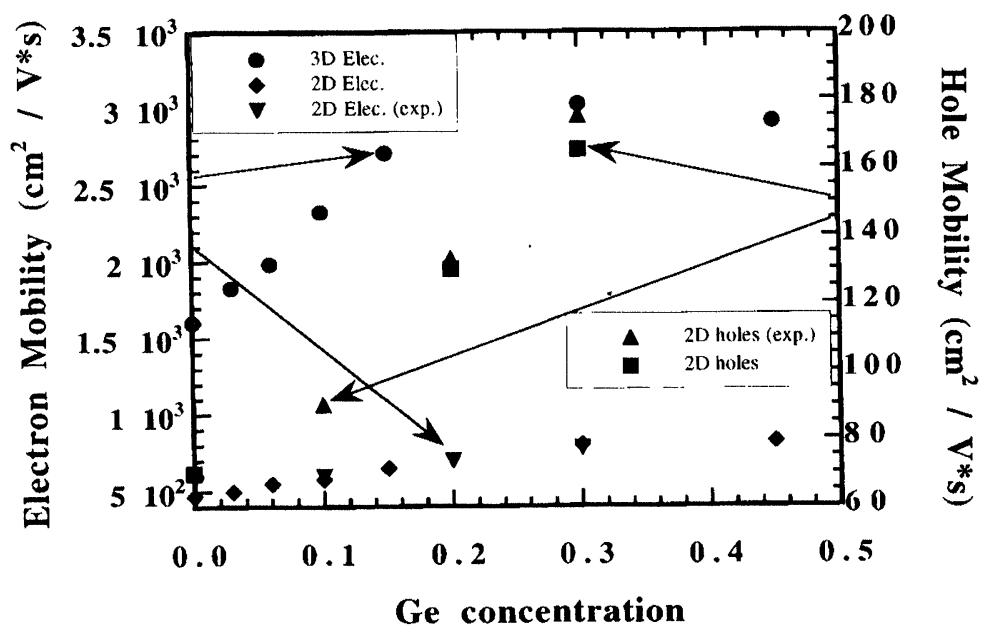

FIGURE 2 Calulated and experimental ([7],[8]) low-field drift mobilities in a strained Si channel for electrons and holes at room temperature for different $\mathrm{Ge}$ concentration in the substrate 


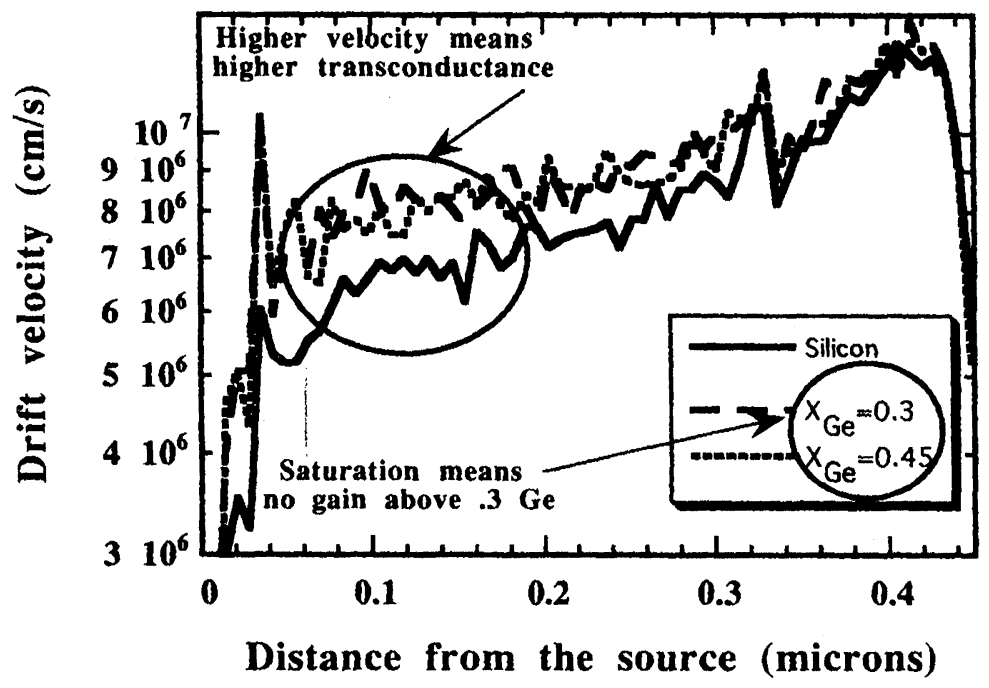

FIGURE 3 Electron drift velocity along the channel for a strained and unstrained Si layer

ess $(\approx 65 \mathrm{meV})$, it is almost totally suppressed and the low-field mobility has increased to its highest value where saturation occurs.

For holes no saturation is observed. The low-field mobility increases almost linearly with the Ge concentration in the substrate. This behavior is due to the different way strain affects the valence band structure. In fact, we do have a splitting in energy between the light and heavy hole bands, but also the strain changes the parallel and perpendicular (Fig. 1) hole effective masses in the channel. These two effects combine together to give a linear increase of low-field mobility with Ge concentration. The results are in good agreement with available experimental data [8].

Finally, an electric field pattern calculated from a MEDICI simulation for a typical nMOSFET with a strained channel has been used in a quasi_2D Monte Carlo simulator and it is found that the electron drift velocity along the channel in strained $\mathrm{Si}$ based devices is higher than in unstrained Si (Fig. 3).

\section{References}

[1] M. M. Rieger and P. Vogl, Electronic-band parameters in strained $S i_{1-x} G e_{x}$ alloys on $S i_{1-x} G e_{x}$ substrates, Phys. Rev. B 48, 14276 (1993).

[2] T Manku and A. Nathan, Energy-band structure for strained p-type $S i_{1-x} G e_{x}$, Phys. Rev. B 43, 12634 (1991).
[3] G. Dresselhaus, A. F. Kip, and C. Kittel, Cyclotron Resonance of Electrons and Holes in Silicon and Germanium Crystals, Phys. Rev. B 98, 368 (1955).

[4] G. E. Pikus and G.L. Bir, Effect of Deformation on the Hole Energy Spectrum of Germanium and Silicon, Sov. Phys.Solid State 1, 1502 (1959).

[5] D. Vasileska-Kafedziska, Ph.D. Thesis, Arizona State University, December 1995.

[6] D. K. Ferry, Semiconductors, New York: Macmillan Publishing Company, 1991.

[7] J. J. Welser, Ph.D. Thesis, Stanford University, December 1994.

[8] J. Hoyt, private communication.

[9] P. Vogl, private communication.

\section{Biographies}

Gabriele F. Formicone is Graduate Student at Arizona State University. He got his BS at the University of Rome "La Sapienza" (Italy). He is currently interested with Monte Carlo simulation of hole and electron trasport properties in strained $\mathrm{Si}$ layers and Medici simulation of $\mathrm{Si}_{1-\mathrm{x}} \mathrm{Ge}_{\mathrm{x}}$ based devices. He spent a summer internship at Motorola ('95).

Dragica Vasileska is faculty research associate in the Center for Solid State Electronics Research at Arizona State University. Her research interests include semiconductor device physics, device simulation, semiconductor transport theory and quantum transport in quasi-two-dimensional structures. She is currently working on transport through quantum dot 
structures. She is a member of the Phi Kappa Phi honor society and American Physical Society.

David K. Ferry is Regent's Professor of Engineering at Arizona State University. His research encompasses transport physics and modeling of submicron devices, particularly the inclusion of quantum effects, and electron-beam lithography of ultra-submicron devices, where his group has fabricated HEMT's with gate lengths as short as $25 \mathrm{~nm}$. He is a fellow of both the American Physical Society and the Institute of Electrical and Electronics Engineers. 

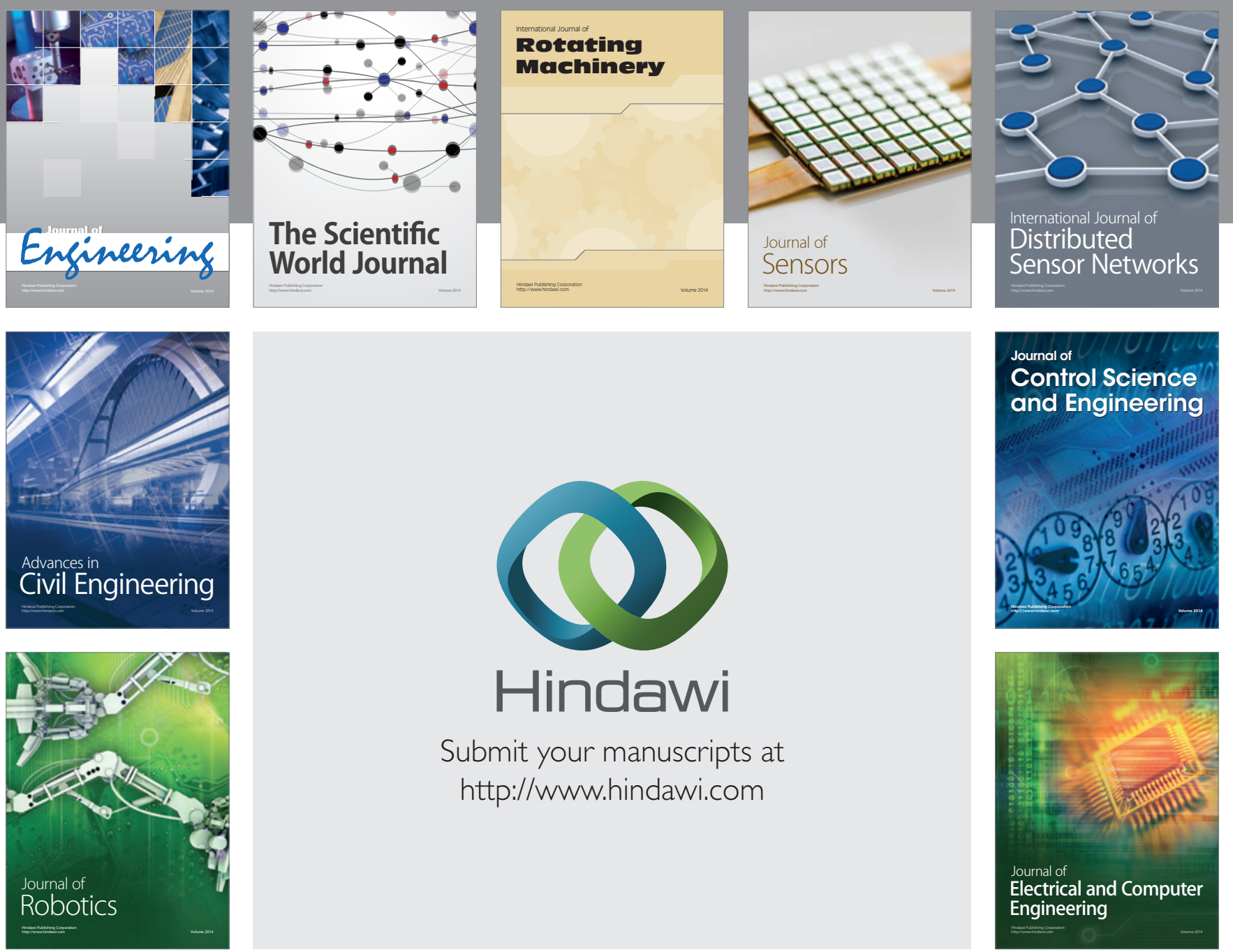

Submit your manuscripts at

http://www.hindawi.com
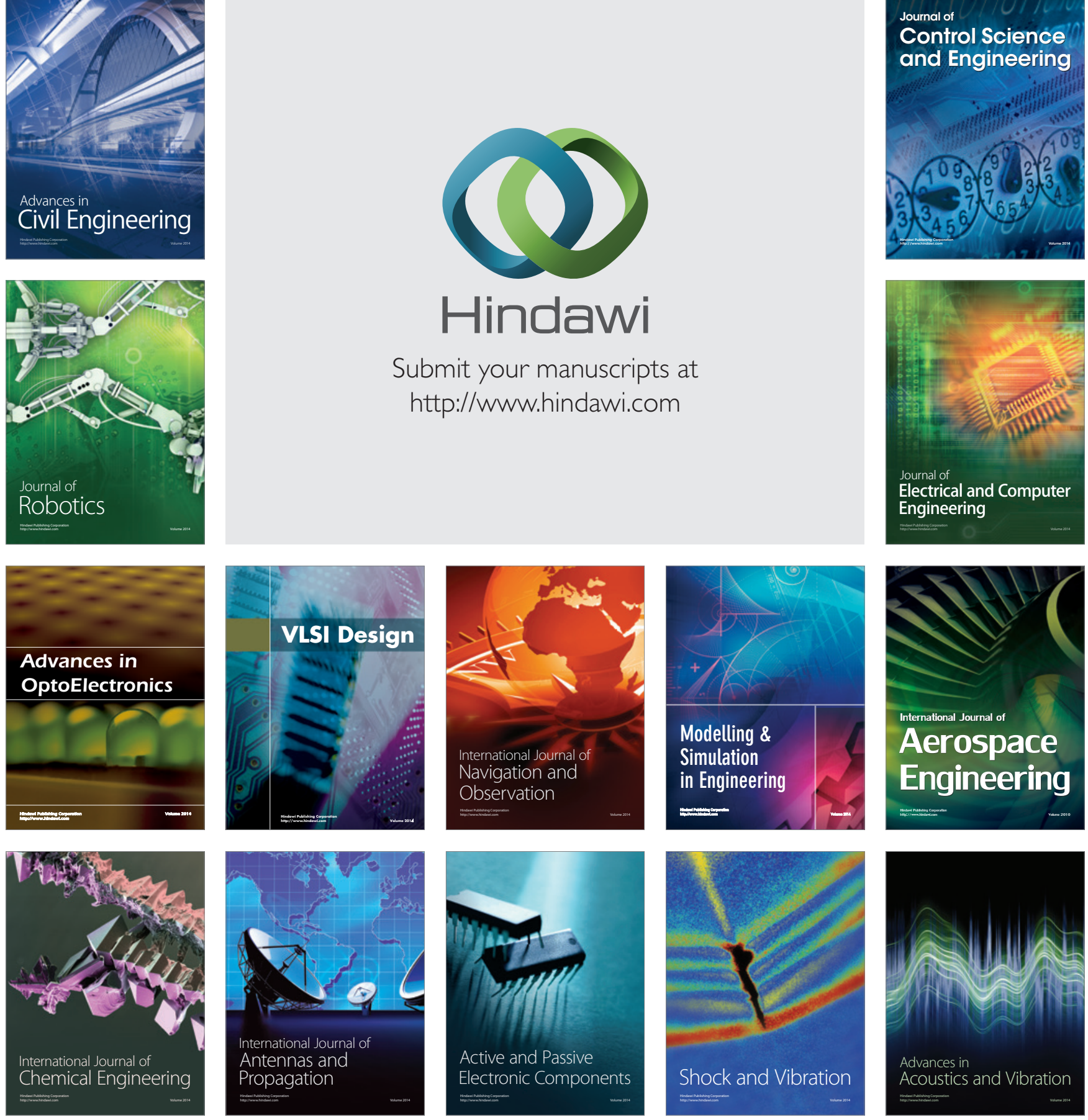